\title{
Relevance Feedback in an Adaptive Space with One-Class SVM for Content- Based Music Retrieval
}

\author{
Gang Chen ${ }^{1}$, Tianjiang Wang ${ }^{1}$, Perfecto Herrera ${ }^{2}$ \\ ${ }^{1}$ Department of Computer Science, Huazhong University of Science and TechnologyWuhan, \\ China \\ ${ }^{2}$ Music Technology Group, Universitat Pompeu Fabra, Barcelona,Spain
}

\begin{abstract}
In this paper, we develop a novel scheme to content-based music retrieval, using relevance feedback with One-class Support vector Machine (SVM). Since one-class SVM only concerns the relevant examples and neglects useful information from irrelevant examples provided by the user, an adaptive space is proposed using both relevant and irrelevant examples. The adaptive space, integrated with one-class SVM, transforms the feature space to a space that would better correspond to the user's needs and specificities. Experimental results of retrieval on a music genre database demonstrate the effectiveness of our approach.
\end{abstract}

\section{Introduction}

Rapid increase in speed and capacity of computers and Internet has created an easy access to enormous music databases. Traditional organization of music files using song's name, singer and other textual tags is not enough for most of the users.

In recent years, to overcome drawbacks recognized in text-based approach, content-based music analysis is getting more attention and a big research effort has been conducted in the area of Content-Based Music Retrieval (CBMR). However, due to the complexity and variability of the music and users' searching intention, it is not realistic to expect a music retrieval system to achieve satisfactory performance consistently for all users' intentions. There are cases where the users are not capable of indicating, in a clear and unambiguous way, their search criteria or what are they expecting to find. In those cases, they can only generate a positive or negative evaluation of the individual music items that have been retrieved by the system. When these conditions are problem held, relevance feedback (RF) is the preferred technique for helping the system to"guess" what is the user looking for.

In fact, RF was first introduced for the retrieval of text documents in [1] and [2]. During retrieval process, the user interacts with the system and selects the relevant examples of the retrieved results, according to his/her subjective judgment. RF technique has been widely used to improve the performance of Content Based Image Retrieval (CBIR) systems [3].

Although efforts on CBMR have been presented in recent years, however, there have been rather few works which have used RF methods for improving their retrieval performance. In related work, Haoshi et al. [4] used relevance feedback for music retrieval which is based on the Tree-structured vector Quantization (TreeQ) method. The approach of the TreeQ method is to train a vector quantizer instead of modeling the music directly. Michael et al. [5] presented an active learning music retrieval system using Support Vector Machine (SVM) and used relevance feedback information to shrink the versions pace of SVM. Seungmin Rho et al. [6] incorporated user's relevance feedback with genetic algorithm to improve retrieval performance and developed a prototype system.

Approaches based on SVM have been widely used and have shown many elegant properties in the relevant feedback area [5][7][8][9]. However, much work using SVM as basic classifier handle the relevance feedback problem as a strict two-class classification problem, with equal treatments on both relevant and irrelevant examples. It is reasonable to assume positive examples to be clustered in a certain way (i.e. being"homogeneous", whereas negative examples may be vary (i.e. difficult to be clustered together), they could belong to any of the remaining classes in the database. If the irrelevant examples are randomly distributed over the feature space, they could be of no help to the classifier which is trying to learn a decision boundary separating the relevant examples from the irrelevant examples. Therefore, we consider 
this retrieval situation is a one-class classification problem rather than a two-class or multi-class classification problem.

However, the irrelevant examples are also highly useful for query refinement since they allow determining the user dislikes. Some valuable information will be discarded if we only concern the relevant examples during the relevance feedback.

In this paper, we proposed an adaptive space that takes into account both relevant and irrelevant examples. It transforms the original feature space into a new one that better corresponds to the user's needs and specificities. In this space, a novel music retrieval scheme which incorporates one-class SVM with relevance feedback is used to improve retrieval performance.

The rest of the paper is organized as follows: Section 2 briefly describes one-class SVM. Section 3 introduces an adaptive space for one-class SVM. Section 4 discusses several experimental results. Finally, we conclude the paper in Section 5.

\section{One-class support vector machine}

One-class SVM was proposed by Scholkopf et al. [10] in 2001 for estimating the support of a highdimensional distribution. The strategy is to map the data into the feature space corresponding to the kernel, and try to use a hyper sphere to describe the data in feature space and put most of the data into the hypersphere. In our context, given training data $X=\left\{x_{1}, x_{2} \ldots x_{i}\right\}, x_{i} \in R^{N}$, where $x$ is a feature vector without any class information, and there are $i \psi$ feature vectors in the $N$-dimensional space $R$. We would like to estimate a function that takes the value +1 - in a"small" region capturing most of the data points, and $\bullet 1$-elsewhere. Formally, we write the function as follows:

$$
f(x)= \begin{cases}+1 & \text { if } x \in S \\ -1 & \text { if } x \in \bar{S}\end{cases}
$$

Where $S \psi$ and $\bar{S} \psi$ are a simple subset of the input space and its complement, respectively. Let $R^{N} \rightarrow F$ be a kernel map that transforms the training data from space $R^{N}$ to a feature space F. To separate the data set from the origin, we need solve the following primal optimization problem:

$$
\begin{aligned}
\min (w, v, \mathcal{\varepsilon}, \rho): & \frac{1}{2}\|w\|^{2}+\frac{1}{v l} \sum_{i=1}^{l} \varepsilon_{i}-\rho \\
\text { subject to: } & w^{T} \cdot \Phi\left(x_{i}\right) \geq \rho-\varepsilon_{i} \\
& \varepsilon_{i} \geq 0, i=1, \ldots, l
\end{aligned}
$$

where $\xi$ is the slack variable, $v \in(0,1)$ is an upper bound on the fraction of outliers and a lower bound on the fraction of support vectors, and $\rho$ is the margin. When we solve the problem, we can obtain w and $\rho$. By introducing Lagrange multipliers $a_{i}$, we can get the following dual problem:

$$
\begin{array}{ll}
\min (a): & \frac{1}{2} a^{T} Q a \\
\text { subject to: } & 0 \leq a_{i} \leq \frac{1}{v l}, i=1, \ldots, l \\
& e^{T} a=v l
\end{array}
$$

where $Q_{i j}=K\left(x_{i}, x_{j}\right)=\Phi\left(x_{i}^{T}\right) . \Phi\left(x_{j}\right)$ is the kernel function which allows much more general decision functions when data are not linearly separable. Frequently used kernel functions are listed in Table 1.

Table 1. SVM Kernels

\begin{tabular}{|c|c|}
\hline Kernel & $k(x, y)$ \\
\hline Linear & $x \cdot y$ \\
\hline Polynomial & $(\gamma(x \cdot y)+\operatorname{cof} 0)^{d}, \gamma>0$ \\
\hline RBF & $\exp \left(-\gamma\|x-y\|^{2}\right), \gamma>0$ \\
\hline Sigmoid & $\tanh (\gamma(x \cdot y)+\operatorname{coe} f 0)$ \\
\hline
\end{tabular}

Eventually, the decision function is:

$$
f(x)=\operatorname{sgn}\left(\sum_{i=1}^{l} a_{i} \cdot K\left(x_{i}, x\right)-\rho\right)
$$

Given a new data point $x$ to classify, the value $f(x)$ is determined by evaluating which side of the hyper-sphere it falls on, in the feature space.

\section{An adaptive space}

Since one-class SVM is only addressed to the distribution of the relevant examples, we may lose much helpful information the user provided about the irrelevant examples. The irrelevant examples are highly useful for query refinement since it allows to determining which music files the user does not want. Our strategy is to transform the original feature space into a new space that would better correspond to the user's needs and specificities, called an adaptive space. The user's intention can be better described in the new adaptive space with user's relevance feedback 
including not only relevant examples, but also irrelevant ones.

The transformation is done by enhancing the importance of those features that help in retrieving relevant examples and reducing the importance of those which do not. Once the importance of each feature is determined, the results are applied to define similarity measures which better correspond to the similarity intended by the user in the current query. Unlike most feature re-weighting approaches which use only the standard deviation from relevant examples, we use a method, inspired by [11], based on a set of statistical characteristics using relevant and irrelevant examples. It uses the following formula to compute the discriminability of its feature component as its weight, which is defined as:

$$
\mathrm{W}_{i}=\frac{1-m / M}{\operatorname{Dev}\left(x_{i}\right)}, m=\left\|y_{i}\right\|
$$

and

$$
\begin{aligned}
& \left(\bar{x}_{i}-\operatorname{Dev}\left(x_{i}\right)\right) \leq y_{i} \leq\left(\bar{x}_{i}+\operatorname{Dev}\left(x_{i}\right)\right) \\
& \bar{x}_{i}=\frac{\sum_{j=1}^{M} x_{i j}}{M} \\
& \operatorname{Dev}\left(x_{i}\right)=\left(\frac{1}{M-1} \sum_{j=1}^{M}\left(x_{i j}-\bar{x}_{i}\right)^{2}\right)^{1 / 2}
\end{aligned}
$$

where $W_{i}$ denotes the weight of the $i$ th feature component. $\bar{x}_{i}$ is the mean value of $i$ th feature component and $\operatorname{Dev}\left(x_{i}\right)$ is its corresponding standard deviation. $M$ represents the total number of relevant music files in database and $m$ represents the total number of irrelevant music files those falls into the range of relevant music files at the $i$ th feature component. The weight indicates the ratio of irrelevant music files located outside the range of relevant music files, which represents the ability of this feature to separate relevant music from irrelevant ones. It only assigns large weights to the feature components which cluster all relevant music files together and scatters all irrelevant music files away from the relevant ones.

Therefore, the new vector in the adaptive space for a feature vector $x_{i}$ is:

$$
x_{i}^{\prime}=x_{i} \cdot W_{i}
$$

\section{Similarity measurement for relevance feedback}

We assume that the user has an ideal query point $q$ that he/she is looking for. A query refinement mechanism is used to improve the estimate of the ideal query point by moving the query point towards the middle of the cluster of relevant music files and away from the irrelevant during the relevance feedback. The sets of relevant music files $M_{r}$ and irrelevant music files $M_{i r}$ specified by the user are used to calculate a new query point $q_{t+1}$ by applying Rocchio's formula [12]:

$$
q_{t+1}=\alpha \cdot q_{t}+\beta+\gamma \cdot \operatorname{mean}\left(M_{r}\right)+\gamma \cdot \operatorname{mean}\left(M_{i r}\right)
$$

where $\alpha+\beta+\gamma=1, \alpha, \beta, \gamma$ control the relative importance of the previous query, the relevant and the irrelevant music files respectively. In our research, we set $\alpha=0.9, \beta=0.8, \gamma=-0.7 \quad$ empirically. This process is iterated across the RF iterations, progressively moving qt towards the optimal query.

For an initial query, the feature vector of the query seed is used as the initial query point. The similarities are calculated based on the Euclidean distances between query point and music files from database.

Once the user interacts with the system and selects the relevant examples from the retrieved results, an adaptive space can be constructed. One-class SVM is then trained by the relevant examples in the adaptive space, and the boundary of the hyper-sphere is learned. Similarities between the query point and the music files in the database are calculated based on the boundary.

The music inside and outside the boundary are treated differently [9]. For the music files $x$ inside the boundary, we rank them based on their Euclidean distances to the query point $q$ in the adaptive space. The distance can be formulated as:

$$
D(x, q)=\sum_{i=1}^{N}\left|x_{i}-q_{i}\right|
$$

We use $M_{-} D$ to represent the maximum Euclidean distance between the music files inside the boundary and the query point.

On the contrary, the music files outside the boundary $x$ are ranked only by their distance to the boundary with reweighed feature components. Intuitionally, the music files relevant to the query may be misclassified and are not enclosed inside the boundary, but typically they are not far away from the boundary. Hence, the likelihood of being relevant depends on the distances to the boundary:

$$
D(x, q)=M_{-} D+\sum_{i=1}^{l} a_{i} \cdot K\left(x_{i}, s_{i}\right)-\rho
$$

where $S_{i}$ is the support vector. 


\section{Experiments and results}

\subsection{Music database and features}

We select one music genre database from Tzanetakis [13]. The database includes a large number of pre-categorized music files. The genre database has the following classes: classical, country, disco, hiphop, jazz, rock, blues, reggae, pop and metal. Each category consists of 100 music files. In total, the selected database has 1000 music files.

In order to estimate more accurately the user's intention, we try to extract as many features as possible, providing a very rich description of every music file in the database. We used a set of 41 features of different kinds, including Temporal, Spectral, Tonal and Rhythm features [14][15][16][17]. Afterward we compute statistics of these features (mean, variance) and normalize them.

\subsection{Experimental setting and procedure}

We present experiments to show the performance of our proposed approach. In a RF based retrieval system, different users may have different opinions and may choose different files as feedback. Even they could determine the same file as relevant or irrelevant. In order to avoid such evaluation problem, we conduct the experiment in a fully automatic way. We assume files in the same class in the database are relevant, otherwise they are considered as irrelevant.

The procedure of the experiment is described as follows:

(1) Treat the ith music category as the relevant and the remaining as irrelevant;

(2) Select an example from the relevant category as a query to launch retrieval, and perform initial query;

(3) Sort the music files in the database according to the

imilarity measurement;

(4) According to the ground truth, automatically label the top $\mathrm{N}$ music files as relevant or irrelevant to simulate user's feedback. After that, these labeled music files are added into the current training data set and removed from the database;

(5) Based on the available training data, use the proposed method to classify the music in the database;

(6) Redo steps 3 to 5 certain times to simulate user feedbacks;

(7) To accumulate statistics, redo steps for each music files in the category. The obtained retrieval performance is averaged respectively;

(8) Redo steps 1 to 7 for each music category in the database and the obtained retrieval performance is averaged.

\subsection{Experimental results}

As the goal of searching music with the same genre is to find as many as relevant music files as possible, therefore, we estimate the performance using recall. Recall for each round is defined as the percentage of true relevant music files retrieved so far among the total relevant ones in the database when top $\mathrm{N}$ are retrieved. We select $\mathrm{N}=20$ for our experiments. We use the LIBSVM [18] and RBF kernel with default parameters to handle one-class SVM and perform the experiments.

With the goal of comparing the search performance with one-class SVM in an adaptive space against oneclass SVM and Two-class SVM in an original space, we conduct the experiment. Figure 1 plots the retrieval results of One-class SVM in an adaptive space (OcSVM Adaptive) using both relevant and irrelevant music files, One-class SVM in the original space (OcSVM Origin) using only relevant music files and Twoclass SVM (Tc-SVM). It is observed that, One-class

SVM in an adaptive space outperform Two-class SVM during the first seven iterations. After seven rounds of relevance feedback, however, Two-class SVM outperforms one-class SVM in an adaptive space. And the average recall over ten iterations for Oc-SVM Adaptive is 0.5823 and Tc-SVM is 0.5378 . Obviously, One-class SVM in an adaptive space performs better than Two-class SVM. They all outperform Oneclass SVM in original space consistently. In the final experiment, we compare the searching performance for different music genre. Table 2 shows the average recall of each category in the database, respectively. It can be seen that, after 4 iterations, the average recall of $54.62 \%$ for ten musical genres is achieved. Rock music gives the worst retrieval precision at $30.26 \%$, because it is easily confused with other genres because of its broad nature. Metal music has the best precision at $83.49 \%$ and is the most predictable because of its special nature. Our results, in terms of error rate per class, seem to roughly follow those reported in [13].

\section{Conclusions}

When performing content-based music retrieval, it is very important to take into account the user's needs and specificities, which, in most of the cases, cannot be defined by means of well-defined music categories. In this case, those needs and search criteria can be indirectly identified by means of RF. In this paper, we proposed a novel One-class SVM based relevance feedback for content-based music retrieval in an 
adaptive space. We validated our system by testing it on a genre database and conduct some performance evaluations. The results show the effectiveness of our approach.

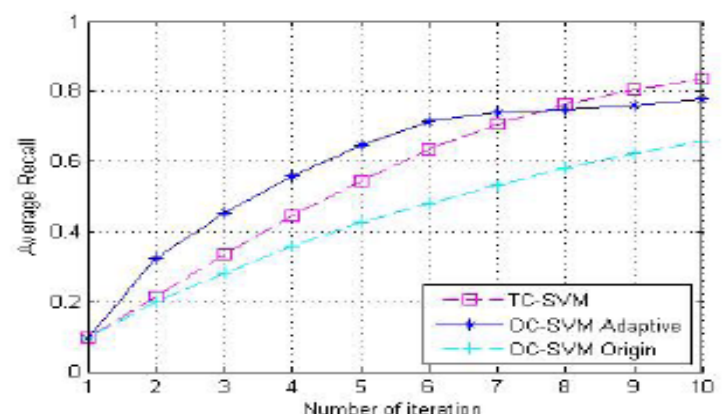

Fig. 1. Comparison of Oc-SVM Adaptive, Tc-SVM and Oc-SVM Origin

Table 2. Genre Database Recall

\begin{tabular}{|c|c|c|c|c|}
\hline \multirow{2}{*}{ Genre } & \multicolumn{4}{|c|}{ Recall } \\
\cline { 2 - 5 } & Iteration1 & Iteration2 & Iteration3 & Iteration4 \\
\hline Blues & 0.1505 & 0.2072 & 0.2651 & 0.3170 \\
Classical & 0.2867 & 0.5337 & 0.6679 & 0.7722 \\
Country & 0.2020 & 0.3103 & 0.4180 & 0.5175 \\
Disco & 0.1712 & 0.2686 & 0.3528 & 0.4328 \\
Hiphop & 0.2922 & 0.4351 & 0.5644 & 0.6711 \\
Jazz & 0.2598 & 0.4149 & 0.5382 & 0.6387 \\
Metal & 0.3775 & 0.5926 & 0.7374 & 0.8349 \\
Pop & 0.2111 & 0.3350 & 0.4531 & 0.5496 \\
Reggae & 0.1593 & 0.2590 & 0.3470 & 0.4263 \\
Rock & 0.1250 & 0.1889 & 0.2465 & 0.3026 \\
\hline
\end{tabular}

\section{Acknowledgment}

The work reported here was done in the Music Technology Group (MTG), Universitat Pompeu Fabra. We want to thank Emila Gomez, Joan Serra, Cyril Laurier and Ferdinand Fuhrmann for their helpful suggestions. We also want to thank the MTG human and technical infrastructure for providing support for this research.

\section{References}

[1] G. Salton, Automatic information organization and retrieval,McGraw-Hill.

[2] C.J. Rijsbergen, Information retrieval, Butterworths, London, 1979.

[3] X.S.Zhou and T.S. Huang, "Relevance feedback in image retrieval: a comprehensive review," Multimedia systems,pp. 536-544, 2003.

[4] K. Hoashi, K. Matsumoto, and N. Inoue, "Personalization of user profiles for content-based music retrieval based on relevance feedback," in International conference on multimedia, 2003, pp. 110 119.
[5] M. I. Mandel, G. E. Poliner, and D. P. W. Ellis, "Support vector machine active learning for music retrieval," Multimedia systems, pp. 3-13, 2006.

[6] S. Rho, E. Hwang, and M. Kim, "Music information retrieval using a GA-based relevance feedback," in International conference on multimedia and ubiquitous engineering, 2007, pp. 739-744.

[7] S.Tong and E.Chang, "Support vector machine active learning for image retrieval," in International conference on multimedia, 2001, pp. 107-118.

[8] L. Wang, Y. Gao, K.L. Chan, P. Xue, and W.Y. Yau, "Retrieval with knowledge-driven kernel design: an approach to improving SVM based CBIR with relevance feedback," in 10th international conference on computer vision, 2005, pp. 1355-1362.

[9] G.D. Guo, A.K. Jain,W.Y. Ma, and H.J. Zhang, "Learning similarity measure for natural image retrieval with relevance feedback," IEEE Transaction neural network, vol. 13, no. 4, pp. 811-820, 2002.

[10] B. Scholkopf, J.C.Platt, J.Shawe-Taylor, A.J.Smola, and R.C. Williamson, "Estimating the support of a high dimensional distribution," Neural computation, vol. 7, no. 13, pp. 1443-1471, 2001.

[11] Y. Wu and A. Zhang, "A feature-reweighting approach for relevance feedback in image retrieval," in International conference on image processing, 2002, pp. $581-584$.

[12] J.J. Rocchio, "Relevance feedback in information retrieval," The SMART retrieval system: experiments in automatic document processing, pp. 313-323, 1971.

[13] Tzanetakis, Essl, and Cook, "Automatic musical genre classification of audio signals," in International conference on music informaiton retrieval, 2001, pp. 205-210.

[14] G. Peeters, "A large set of audio features for sound description (similarity and classification) in the CUIDADO project".

[15] Skovenborg, E. Nielsen, and S.H., "Evaluation of different loudness models with music and speech material," in AES 117th convention, San Francisco, CA, USA, 2004.

[16] S. Streich, Music complexity: a multi-faceted description of audio content, Ph.D. thesis, UPF, Barcelona, Spain, 2007.

[17] E. Gomez, Tonal Description of music audio signals, Ph.D. thesis, UPF, Barcelona, Spain, 2006.

[18] C.C. Chang and C.J. Lin, "Libsvm : a library for 
support

vector

machines, 2001,”

http://www.csie.ntu.edu.tw/ cjlin/libsvm. 